\title{
Distribuição de casos novos da hanseníase em um estado do Nordeste
}

\author{
Distribution of new cases of leprosy in a northeast state \\ Distribución de nuevos casos de lepra en un estado del noreste
}

Recebido: 18/12/2021 | Revisado: 27/12/2021 | Aceito: 03/01/2022 | Publicado: 04/01/2022

Robert Lincoln Barros Melo
ORCID: https://orcid.org/0000-0002-3430-4411
Universidade Federal de Alagoas, Brasil
E-mail: robert.lincoln.b@ gmail.com
Amuzza Aylla Pereira dos Santos
ORCID: https://orcid.org/0000-0001-6299-7190
Universidade Federal de Alagoas, Brasil
E-mail: amuzzasantos@ bol.com.br
Isabel Comassetto
ORCID: https://orcid.org/0000-0002-2389-9384
Universidade Federal de Alagoas, Brasil
E-mail: isabelcomassetto@gmail.com
Valéria Bezerra Santos
ORCID: https://orcid.org/0000-0002-3026-9823
Secretaria de Estado da Saúde de Alagoas, Brasil
E-mail: valeria.santos67@gmail.com
Alice Correia Barros
ORCID: https://orcid.org/0000-0002-2627-7185
Universidade Federal de Alagoas, Brasil
E-mail: alicebarros.enf@ gmail.com
Thaís Honório Lins Bernardo
ORCID: https://orcid.org/0000-0002-8058-8400
Universidade Federal de Alagoas, Brasil
E-mail:thais.bernardo@eenf.ufal.br
Wanderlei Barbosa dos Santos
ORCID: https://orcid.org/0000-0001-9813-8857
Universidade Federal de Alagoas, Brasil
E-mail: wanderlei.santos@esenfar.ufal.br
José Augustinho Mendes Santos
ORCID: https://orcid.org/0000-0002-1570-4102
Universidade Federal de Alagoas, Brasil
E-mail: augustinhomendes1@gmail.com

\section{Resumo}

Objetivo: analisar a distribuição de casos novos da hanseníase em um estado do Nordeste entre os anos de 2008 e 2017. Métodos: estudo epidemiológico descritivo, retrospectivo de abordagem quantitativa, realizada a partir dos dados secundários do SINAN; população composta pelos casos novos notificados entre 2008 a 2017. Realizou-se análise descritiva, buscando-se esquematizar a variabilidade dos dados entre si. Resultados: Seguindo a mensuração dos dados, eles foram apresentados de forma descritivas para as frequências absoluta e relativa. Foram notificados 3.542 casos no período. As variáveis estudadas foram sexo, faixa etária, ano de diagnóstico e características clínicas. Do total 51,8\% eram do sexo feminino, e a faixa etária concentrou-se entre 30 e 39 e anos (18,4\%). A forma clínica mais prevalente foi a multibacilar. A taxa média de detecção foi de 21,23 casos novos para cada 100 mil habitantes. Conclusão: o estudo evidencia a necessidade de intensificação e concretização da vigilância em hanseníase.

Palavras-chave: Hanseníase; Doenças negligenciadas; Epidemiologia; Saúde pública.

\begin{abstract}
Objective: to analyze the distribution of new leprosy cases in a state of northeast between the years 2008 and 2017. Methods: descriptive, retrospective epidemiological study with a quantitative approach, carried out using secondary data from SINAN; population composed of new cases notified between 2008 to 2017. A descriptive analysis was carried out, seeking to outline the variability of the data among themselves. Results: Following the measurement of the data, they were presented in a descriptive way for the absolute and relative frequencies. 3,542 cases were reported in the period. The variables studied were sex, age group, year of diagnosis and clinical characteristics. Of the total, $51.8 \%$ were female, and the age group was concentrated between 30 and 39 years old (18.4\%). The most prevalent clinical form was multibacillary. The average detection rate was 21.23 new cases for every 100 thousand inhabitants. Conclusion: the study highlights the need for intensification and implementation of leprosy surveillance.
\end{abstract}

Keywords: Leprosy; Neglected diseases; Epidemiology; Public health. 


\section{Resumen}

Objetivo: analizar la distribución de nuevos casos de lepra en un estado de noreste entre los años 2008 y 2017. Métodos: estudio epidemiológico descriptivo, retrospectivo con enfoque cuantitativo, realizado con datos secundarios del SINAN; población compuesta por nuevos casos notificados entre 2008 y 2017. Se realizó un análisis descriptivo, buscando delinear la variabilidad de los datos entre ellos. Resultados: Luego de la medición de los datos, se presentan de manera descriptiva para las frecuencias absolutas y relativas. Se notificaron 3.542 casos en el período. As variáveis estudadas foram sexo, faixa etária, ano de diagnóstico e características clínicas. Do total, 51,8\% eram mulheres, e a faixa etária concentrava-se entre 30 e 39 anos $(18,4 \%)$. La forma clínica más prevalente fue la multibacilar. La tasa de detección promedio fue de 21,23 casos nuevos por cada 100 mil habitantes. Conclusión: el estudio destaca la necesidad de intensificar e implementar la vigilancia de la lepra.

Palabras clave: Lepra; Enfermedades desatendidas; Epidemiología; Salud pública.

\section{Introdução}

A hanseníase é uma doença infecciosa, transmissível e de caráter crônico, de notificação compulsória, negligenciada e que ainda persiste como um grave problema de saúde pública no Brasil. É causada pelo agente etiológico Mycobacterium leprae, conhecido por Bacilo de Hansen e possui um período de incubação prolongado em torno de dois a sete anos. (Brasil, 2017; Goes, 2016; Pires et al., 2012). A doença atinge pessoas de qualquer sexo ou faixa etária, podendo apresentar evolução lenta e progressiva e, quando não tratada, pode causar deformidades e incapacidades físicas, muitas vezes irreversíveis (Brasil, 2020; Brasil, 2017; Brasil, 2016a).

Essa bactéria manifesta-se na maioria das vezes na população de baixa renda, em ambos os sexos e faixa etária, provocando o comprometimento no tecido cutâneo e nervos periféricos podendo causar incapacidades físicas e deformidades quando não diagnosticada precocemente (Brasil, 2017; Goes, 2016; Pires et al., 2012).

É uma doença negligenciada por está diretamente relacionada sua detecção as condições socioeconômicas e culturais da população acometida. Dessa forma, as condições de habitação, os baixos índices de escolaridade e os movimentos migratórios podem facilitar a disseminação da doença, bem como, a aglomeração de pessoas e o contato prolongado com doentes multibacilares não tratados (Souza, Luna \& Magalhães, 2019).

No mundo, foram reportados à Organização Mundial da Saúde (OMS) 208.619 casos novos da doença em 2018. Desses, 30.957 ocorreram na região das Américas e 28.660 (92,6\% do total das Américas) foram notificados no Brasil. Diante desse cenário, o Brasil é classificado como um país de alta carga para a doença, ocupando o segundo lugar na relação de países com maior número de casos no mundo, estando atrás apenas da Índia (WHO, 2019). Dados preliminares de 2019 mostram que o Brasil diagnosticou 23.612 casos novos de hanseníase, sendo 1.319 (5,6\%) em menores de 15 anos (Brasil, 2020).

A hanseníase faz parte da Lista Nacional de Notificação Compulsória de doenças (Portaria de Consolidação MS/GM $\mathrm{n}^{\circ}$ 4, de 28 de setembro de 2017), e, portanto, é obrigatório que os profissionais de saúde reportem os casos da doença no Sistema de Informação de Agravos de Notificação (SINAN). A análise dos dados do sistema é fundamental para identificar diferentes padrões de ocorrência da doença, as áreas de maior vulnerabilidade e as fragilidades na vigilância dessa endemia no Brasil. A produção e divulgação de informação é importante na medida em que permite orientar a tomada de decisão e trazer um olhar mais crítico ao sistema, de forma a identificar inconsistências que interfiram na qualidade da informação (Brasil, 2020).

Neste sentido, é de extrema importância que todas as ações da Atenção Primária estejam conectadas com a Vigilância em Saúde, para que ocorra a análise da situação de saúde da população e planejamento das práticas adequadas para problemas existentes de maneira eficaz. Uma das ferramentas que pode alicerçar a junção da assistência e vigilância é o SINAN, cujo objetivo é formular e avaliar os programas de saúde, auxiliando para as decisões e contribuindo para melhoria dos serviços de saúde (Brasil, 2010). 
Com isso, e, por se tratar de uma doença reemergente por sua vez ainda muito negligenciada nas formas de detecção e ao realizar as notificações, surgiu o interesse em compreender a distribuição de casos novos da hanseníase, para subsidiar processos de elaboração, execução e implementação de políticas públicas para enfretamento da hanseníase.

Desta forma, o estudo traz como objetivo analisar a distribuição de casos novos da hanseníase em um estado do Nordeste entre os anos de 2008 e 2017.

\section{Metodologia}

Trata-se de um estudo de caráter epidemiológico descritivo, retrospectivo de abordagem quantitativa, realizada a partir dos dados secundários do SINAN. Os dados foram coletados no primeiro semestre de 2020, a partir das informações disponíveis nos sites TABNET/DATASUS do Ministério da Saúde. Delimitou-se a amostra ao período de 2008 a 2017. Os dados oficiais de 2018 e 2019 ainda não foram disponibilizados pelo Ministério da Saúde.

As variáveis estudadas foram sexo, faixa etária, ano de diagnóstico e características clínicas (tipo de entrada, tipo de saída, tipo de encerramento, número de lesões cutâneas, forma clínica, classificação operacional, número de nervos afetados e avaliação do grau de incapacidade física). A classificação operacional foi definida de acordo com a Organização Mundial da Saúde (OMS) sendo paucibacilar até cinco lesões e como multibacilar quando o paciente é acometido com mais de cinco lesões (OMS, 2009).

A análise foi realizada através de estatística descritiva, respeitando-se os aspectos éticos da Resolução № 510/16 do Conselho Nacional de Saúde (Brasil, 2016b), que dispõem sobre as normas aplicáveis as pesquisas de acesso público, cujas informações não expõem os participantes envolvidos, excluindo qualquer possibilidade de identificação individual, portanto não acarretando riscos à população em estudo.

Posteriormente, os dados foram submetidos à análise descritiva, buscando-se esquematizar a variabilidade dos dados entre si. Seguindo a mensuração dos dados, eles foram apresentados de forma descritivas para as frequências absoluta e relativa.

\section{Resultados}

No período de 2008 a 2017, foram diagnosticados 3.542 casos novos de hanseníase, o que equivale a uma taxa média de detecção de 21,23 casos novos para cada 100 mil habitantes.

Ao analisar o período no que se refere à distribuição dos casos novos notificados, observa-se uma oscilação da taxa de detecção de 12,49 (2008) a 14,17 (2012), porém há uma queda nos anos de 2016 e 2017, com uma taxa de detecção de 8,22 e 8,04, respectivamente. Entre estes, 1.835 casos novos ocorreram no sexo feminino, correspondente a 51,8\% do total (Tabela 1).

Tabela 1: Distribuição de casos novos de hanseníase, segundo sexo, Alagoas, 2008 a 2017.

\begin{tabular}{ccc}
\hline Sexo & $\mathbf{N}^{\mathbf{0}}$ & $\boldsymbol{\%}$ \\
\hline Feminino & 1835 & 51,8 \\
Masculino & 1706 & 48,1 \\
Ignorado & 1 & 0,1 \\
\hline
\end{tabular}

Fonte: Ministério da Saúde/SVS - Sistema de Informação de Agravos de Notificação - SINAN Net.

Em relação à faixa etária (em anos), o maior número de casos concentra-se entre 30 e 39 e anos (18,4\%), seguido da faixa etária de 40 e 49 anos, com 17,4\% de casos novos. De acordo com os dados levantados quanto à faixa etária no período estudado, o estudo revela que a hanseníase atinge predominantemente os adultos (Tabela 2). 
Tabela 2: Distribuição de casos novos de hanseníase, segundo faixa etária, Alagoas, 2008 a 2017.

\begin{tabular}{ccc}
\hline Faixa etária (em anos) & $\mathbf{N}^{\mathbf{0}}$ & $\mathbf{\%}$ \\
\hline 01 a 04 & 04 & 0,2 \\
05 a 09 & 72 & 2,0 \\
10 a 14 & 149 & 4,2 \\
$15-19$ & 190 & 5,3 \\
$20-29$ & 561 & 15,8 \\
$30-39$ & 654 & 18,4 \\
$40-49$ & 617 & 17,4 \\
$50-59$ & 601 & 16,9 \\
$60-69$ & 436 & 12,3 \\
$70-79$ & 202 & 6,0 \\
80 anos e mais & 56 & 1,5 \\
\hline
\end{tabular}

Fonte: Ministério da Saúde/SVS - Sistema de Informação de Agravos de Notificação - SINAN Net.

No que se refere ao modo de detecção, em Alagoas, observa-se uma redução de $44 \%$ na proporção dos modos que evidenciam a vigilância passiva (encaminhamento e demanda espontânea) quando analisado os anos de 2012 e 2017. Além disso, no período analisado, nota-se também uma redução de 27,6\% dos modos de detecção de vigilância ativa (exame de coletividade e exame de contatos). Dentre os casos novos diagnosticados no período estudado, 55,6\% foram detectados por encaminhamento e $4,8 \%$ por exame de contatos.

Quanto ao número de lesões cutâneas, apenas 1,4\% não apresentavam nenhuma lesão no diagnóstico e 39,3\% apresentavam entre 2 e 5 lesões cutâneas. Todavia, 15,0\% dos casos notificados apresentavam, no diagnóstico, mais de 5 lesões e 15,3\%dos casos estavam não assinalados (em branco), observando uma possível falha no preenchimento da ficha de notificação.

Ao analisar a distribuição dos casos novos, segundo a classificação operacional diagnóstico, 45,6\% dos casos foram classificados como paucibacilar e 54,4\% dos casos como multibacilar.

Em relação as formas clínicas da hanseníase, observou-se que a maior parte dos casos se apresenta com a forma dimorfa da doença, com prevalência maior no sexo masculino de 13,2\%. A forma virchowiana também apresentou em nossa série maior acometimento no sexo masculino (10,8\%). As demais formas clínicas foram mais prevalentes no sexo feminino (26,5\%), distribuídas nas formas indeterminada $(12,1 \%)$ e tuberculóide $(14,4 \%)$ dos casos.

Ao analisar a quantidade de nervos afetados, identificou-se que 3,7\% dos casos tiveram menor e igual a 5 nervos afetados e 0,2 maior que 5 nervos afetados. Com relação ao grau de incapacidade, observou-se que os casos apresentaram, ao diagnóstico, Grau 0 de incapacidade física (60,2\%), seguido pelos que apresentaram Grau I (23,0\%) e Grau II (7,5\%).

Quanto ao tipo de saída, 55,7\% dos casos novos evoluíram para cura; 1,4\% corresponderam a óbitos, 4,3\% de abandono e transferidos representaram 7,3\%. Na maioria dos casos, houve a evolução sem incapacidade física durante o tratamento $/(80,4 \%)$. No entanto, foram classificados como Graus I e II ao final do tratamento, respectivamente, $8,9 \%$ e $2,8 \%$ dos casos.

\section{Discussão}

É sabido que dentre as doenças infecciosas, a hanseníase é considerada uma das principais causas de incapacidades físicas, em razão do seu potencial de causar lesões neurais.

Essas incapacidades têm sido ponderadas pelo estigma e pela discriminação da sociedade, com tudo cabe a importância de sempre procurar o serviço de saúde assim que detectadas as primeiras lesões, para que o serviço possa encaminhar em tempo hábil, pois o estudo mostrou que a forma de detecção em maior quantidade deu-se ao modo de encaminhamento quando o profissional examinou o paciente. Um estudo relatou que uma das formas mais eficazes de 
determinar se o diagnóstico tem sido precoce é identificando a presença de incapacidades físicas no momento da consulta e avaliação (Pereira et al., 2011; Façanha et al., 2020).

No âmbito nacional, o Ministério da Saúde elaborou a Estratégia Nacional para Enfrentamento da Hanseníase 20192022. O documento, que está em fase de publicação, tem como visão um Brasil sem hanseníase. A Estratégia Nacional se baseia na Estratégia Global e tem como objetivo geral reduzir a carga da doença no país ao fim de 2022, para que se consiga atingir as metas é preciso que o diagnóstico precoce possa ser realizado e que o portador da lesão consiga tratamento e seguimento de qualidade para que sua recuperação seja sem sequelas que a doença pode transmitir. Com isso as metas que precisam ser cumpridas serão: redução das taxas em crianças e adultos com grau 2 de incapacidade física e implantação em todas as Unidades da Federação canais para registro de práticas discriminatórias às pessoas acometidas pela hanseníase e seus familiares (Brasil, 2019).

Ë importante destacar que o estudo apresentou alta incidência para hanseníase em indivíduos do sexo feminino, contrapondo o estudo de Almeida et al., (2012) que afirma que os fatores construtores da identidade masculina condicionam aspectos importantes da epidemiologia da hanseníase e de diversas outras moléstias, sendo elas infecciosas ou não. Considerando o cuidado em saúde do gênero feminino, pode-se assumir como características, observação e toque em seu corpo, maior preocupação com aspectos relacionados ao seu corpo e sua saúde, assim como maior tempo para acesso aos serviços de saúde. Embora não existam aspectos fisiológicos identificados que expliquem tal achado, sugere-se que a maior preocupação da mulher com a estética e a saúde induza a procura aos serviços de saúde, o que facilitaria o acesso ao diagnóstico (Romão \& Mazzoni, 2013; Sales et al., 2020).

A hanseníase impacta no cotidiano dos indivíduos afetados, através de preconceito, sofrimento, problemas estéticos e emocionais. Infelizmente, em nossa sociedade as mulheres já enfrentam preconceitos e desigualdades de gênero, assim, podemos considerar os efeitos da doença ainda mais graves neste extrato da população (Gonçalves et al., 2018).

$\mathrm{Na}$ análise segundo faixa etária, a hanseníase tem afetado a população economicamente ativa influenciando nas atividades produtivas devido às reações hansênicas e incapacidades físicas. Neste estudo, 18,4\% dos indivíduos diagnosticados possuíam idade entre 30 e 39 anos, e este dado é semelhante a pesquisa realizada no município de Timon - MA (Barbosa et al., 2014). A hanseníase atingiu substancialmente menos crianças e adolescentes do que adultos. Indica-se que, embora seja baixa a incidência da hanseníase em crianças, esse é um indicador sensível da transmissão da doença e indica que a criança teve um contato com o portador da doença sem tratamento. Recomenda-se, portanto, que, em áreas de alta ou baixa endemia, seja feito o levantamento do contato em uma família que possui crianças com hanseníase para se detectar precocemente a doença (Hazarika et al., 2016).

No que tange as formas clínicas, observou-se maior frequências nas formas dimorfa e tuberculóide, totalizando em média $(44,9 \%)$ dos casos. Além disso, vale salientar que as formas dimorfa e virchowiana foram mais prevalentes no sexo masculino $24,1 \%$.

Infere-se que, dentre as formas clínicas, a dimorfa é a mais transmissível e potencialmente incapacitante. Comprovase que os indivíduos MB são mais vulneráveis à hanseníase devido à resposta imunitária celular não ser eficaz, adotando-se os microrganismos mecanismos de evasão frente à resposta imunológica (Queirós et al., 2016).

Os achados deste estudo corroboraram com a pesquisa de Oliveira et al., (2018), realizada por meio de pesquisa de dados no SINAN nacional em 2015, que relatou encontrar a forma dimorfa em aproximadamente $40 \%$ dos casos notificados.

Quando analisados os dados referentes a classificação operacional do diagnóstico, associada ao sexo, observou-se que houve maior frequência da forma multibacilar no sexo masculino $(33,6 \%)$ e a forma paucibacilar com maior frequência no sexo feminino $(31,0 \%)$ dos casos novos. Ao passo que temos mais casos MB, pode-se pensar em manutenção da endemia, quando os indivíduos mais vulneráveis são afetados e na existência de um sistema de atendimento primário e epidemiológico 
precários o que facilita a perpetuação da doença. Por outro lado, cabe salientar que indivíduos diagnosticados nas formas PB possuem pouco comprometimento da qualidade de vida. Isto leva a sugerir que, quanto mais precoce é o diagnóstico e o tratamento, melhor será a qualidade de vida do paciente (Oliveira et al., 2014).

As incapacidades físicas na hanseníase são importantes sinalizadores do diagnóstico tardio e manifestam-se por perda de sensibilidade protetora, diminuição da força muscular e/ou surgimento de deformidades visíveis. Ocorrem nas mãos e/ou nos pés e/ou nos olhos. Classifica-se o caso de hanseníase como incapacidade grau 0, quando a força muscular e a sensibilidade desses segmentos estão preservadas; grau 1, quando há diminuição da força muscular e/ou diminuição de sensibilidade; e grau 2, quando há deformidade visível nas mãos e/ou pés e/ou olhos (Brasil, 2020).

As deformidades visíveis são uma das principais causas do estigma e do isolamento de pessoas na sociedade. Ao analisar-se os dados observa-se que esta situação afeta a vida de milhares de brasileiros, porque compromete mecanismos de defesa, como a capacidade de sentir dor, a visão e o tato, tornando-os mais vulneráveis aos riscos de acidentes, queimaduras, feridas, infecções e amputações, entre outros (Brasil, 2017).

A vigilância ao indivíduo com hanseníase deve ser realizada por meio da busca ativa e/ou passiva dos casos, sendo a mais comum àquela em cujos casos são detectados na população presente nos serviços de saúde ou ainda por encaminhamentos realizados por serviços pertencentes à rede de atendimento, realidade esta encontrada no presente estudo, no qual os encaminhamentos foi a forma de descoberta precoce do agravo (Brasil, 2020).

Apesar da predominância das notificações passivas dos casos de hanseníase, aspectos referentes à busca ativa dos casos devem ser incentivados com ampla divulgação dos sinais e sintomas à população, com ações de educação em saúde que abordem a temática, sobretudo em regiões onde há alta incidência do agravo. Atenção especial deve ser dada às populações vulneráveis como os contatos de indivíduos infectados e grupos específicos vivendo em aglomerados, como as pessoas privadas de liberdade (Mistura et al., 2015).

O diagnóstico de hanseníase deve ser exposto ao paciente com cautela, enfatizando a característica de cura em caso de adesão ao tratamento. É importante ainda o apoio da rede de serviços de saúde em caso de impacto psicológico, tanto a quem adoeceu quanto aos familiares ou pessoas de sua rede social, com acolhimento da equipe de saúde e de profissionais especializados no auxílio quanto a aceitação do problema, superação das dificuldades e incentivo à adesão aos tratamentos. Essa atenção deve ser oferecida no momento do diagnóstico, bem como no decorrer do tratamento da doença e, se necessária, após a alta (Brasil, 2017).

Neste sentido, as ações devem buscar compreender a execução de práticas realizadas individual ou coletivamente e podem ser orientadas pela equipe multiprofissional, com o intuito de estimular o empoderamento dos sujeitos enquanto protagonistas de suas demandas de saúde. As ações que as pessoas executam diariamente para prevenir, controlar ou reduzir o impacto das condições crônicas caracterizam o autocuidado, já as intervenções da equipe de saúde que ajudam as pessoas a se qualificarem são denominadas autocuidado apoiado.

O presente estudo apresentou algumas limitações devido a indisponibilidade de alguns dados no SINAN NET, dificultando uma avaliação diagnóstica completa e fiel à realidade.

\section{Considerações Finais}

A análise dos casos de hanseníase notificados nos anos de 2008 a 2017 mostrou maior número de casos em 2012; e que neste período predominaram as notificações do gênero feminino, sobretudo na faixa etária entre 30 e 39 anos e lesões/incapacidades relacionadas às formas tuberculóide e dimorfa.

Além disso, pode-se observar que ao decorrer dos anos, houve uma redução expressiva no número de casos da hanseníase em Alagoas, o que demonstra que a operacionalização de políticas de controle da doença estão se tornando eficazes 
possibilitando assim inferir que o diagnóstico tem se tornado precoce e as estratégias de prevenção e controle da doença mais eficaz pela notória redução nos números de casos.

Ressalta-se a necessidade de intensificação e concretização da vigilância em hanseníase, o que possibilita as ações de promoção em saúde e a avaliação das intervenções relacionadas a essa doença negligenciada, pois quando o serviço é efetivo o paciente consegue ser diagnosticado, tratado e tem seu seguimento garantido no serviço, evoluindo assim para cura e reduzindo as sequelas desta doença. Por fim, cabe ressaltar a importância de estudos como esse para nortear gestores e os profissionais de saúde, especificamente aos que atuam no planejamento e gestão de políticas de saúde para prevenção e controle de doenças e agravos transmissíveis.

\section{Referências}

Almeida, M. G. et al. (2012). Saúde e masculinidade: uma calamidade negligenciada. In: Anais do IV Congresso Internacional de Estudos sobre Diversidade Sexual e de Gênero da ABEH. 135-6.

Barbosa, D.R.M., Araújo, A.A., Damaceno, J.C.F., Almeida, M.G. \& Santos, A.G. (2014). Perfil Epidemiológico da Hanseníase em Cidade Hiperendêmica do Maranhão, 2005-2012. Revista Rede de Cuidados em Saúde. 8(1):1-13.

Façanha, A. T. F., Conceição, H. N. da, Oliveira, M. R., Borges, L. V. A., Pereira, B. M., Moura, L. R. P., Chaves, T. S., Lima, D. L. dos S., \& Câmara, J. T. (2020). Análise das incapacidades físicas por hanseníase em uma cidade do interior do Maranhão, Brasil. Research, Society and Development, 9(2): 1-19.

Goes, F. S. (2016). Marcadores sorológicos moleculares para contatos de Hanseníase em menores de 15 anos. Alagoas: Ufal.

Gonçalves, M., Prado, M. A. R., Silva, S. S., Santos, K. S., Araujo, P. N. \& Fortuna, C. M. (2018). Trabalho e hanseníase: as mulheres em suas dores, lutas e labutas. Rev Bras Enferm 71(Suppl 1):660-7.

Hazarika, D., Pawar, M. K. \& Dowerah, E. (2017). A Prospective Study of Clinic-Histopathological Correlation among Leprosy Patients Attending a Tertiary Referral Centre in Assam, in This Post Elimination Era. Int J Health Sci Educ, 7(4):148-53.

Ministério da Saúde (2010). Roteiro para uso do sistema de informação de agravos de notificação - Sinan NET HANSENÍASE. Brasília, DF. http://portalsinan.saude.gov.br/images/documentos/Agravos/Hanseniase/Manual_tabulacao_dos_indicadores_de_hanseniase.pdf

Ministério da Saúde (2016a). Diretrizes para vigilância, atenção e eliminação da hanseníase como problema de saúde pública. Brasília, DF. https://portalarquivos2.saude.gov.br/images/pdf/2016/fevereiro/04/diretrizes-eliminacao-hanseniase-4fev16-web.pdf

Ministério da Saúde (2016b) Resolução Nº 510 do Conselho Nacional de Saúde, de 01 de abril de 2016 (BR). Dispõe sobre as normas aplicáveis a pesquisas em Ciências Humanas e Sociais cujos procedimentos metodológicos envolvam a utilização de dados diretamente obtidos com os participantes ou de informações identificáveis ou que possam acarretar riscos maiores do que os existentes na vida cotidiana. Diário Oficial da União. http://conselho.saude.gov.br/resolucoes/2016/Reso510.pdf.

Ministério da Saúde (2017). Guia Prático sobre a hanseníase. Brasília, DF: https://portalarquivos2.saude.gov.br/images/pdf/2017/novembro/22/Guia-Praticode-Hanseniase-WEB.pdf

Ministério da Saúde (2019). Estratégia Nacional para Enfrentamento da Hanseníase 2019-2022. Brasília, DF. http://www.aids.gov.br/ptbr/pub/2020/estrategia-nacional-para-enfrentamento-da-hanseniase-2019-2022

Ministério da Saúde (2020). Boletim Epidemiológico Especial. Brasília, DF. https://antigo.saude.gov.br/images/pdf/2020/janeiro/31/Boletim-hanseniase-2020web.pdf

Mistura, C., Silva, R. C. C., Vieira, M. C. A., Lira, M. O. S. C., Jacobi, C. S. \& Carvalho, M. F. A. (2015). Leprosy prevention in prison units: reporting the experience of extension activities. Journal of Nursing UFPE. 9(5):7967-7973.

Oliveira, A. K., Freire, F. F. S. \& Nascimento, M. R. F. (2018). Incidência e Perfil Clínico Epidemiológico da Hanseníase no Brasil. Revista Científica da FASETE. 1: 95-108.

Oliveira, J. C. F., Leão, A. M. M. \& Britto, F. V. S. (2014). Análise do perfil epidemiológico da hanseníase em Maricá, Rio de Janeiro: uma contribuição da enfermagem. Rev. de Enfermagem da UERJ. 22(6):815-21.

Pereira, E. V. E., Nogueira, L. T., Machado, H. A. S., Lima, L. A. N. \& Ramos, C. H. M. (2011) Perfil epidemiológico da hanseníase no município de Teresina, no período de 2001-2008. An Bras de Dermatologia.;86(2):235-240.

Pires, A. G. A., Malcher, C. M. S. R., Júnior, J. M. C. A., Albuquerque, T. G., Corrêa, I. R. S. \& Daxbacher, E. L. R. (2012). Hanseníase em menores de 15 anos: a importância do exame de contato. Rev Paul de Pediatr. 30(2):292-29.

Queirós, M. I., Junior, A. N. R., Alencar, C. H. M., Monteiro, L. D., Sena, A. L. \& Barbosa, J. C. (2016). Clinical and epidemiological profile of leprosy patients attended at Ceará, 2007-2011. An Bras Dermatol. 91(3):311-7.

Romão, R. E. \& Mazzoni, A. M. (2013). Perfil epidemiológico da hanseníase no município de Guarulhos, SP. Revista de Epidemiologia e Controle de Infecção. 3(1):22-27. 
Research, Society and Development, v. 11, n. 1, e15011124917, 2022

(CC BY 4.0) | ISSN 2525-3409 | DOI: http://dx.doi.org/10.33448/rsd-v11i1.24917

Souza, C. D. F., Luna, C. F. \& Magalhães, M. A. F. M. (2019). Transmissão da hanseníase na Bahia, 2001-2015: modelagem a partir de regressão por pontos de inflexão e estatística de varredura espacial. Epidemiologia e Serviços de Saúde. 28(1):1-13.

Sales, B. N., Sousa, G. O., Machado, R. S., Rocha, G. M. de M., \& Oliveira, G. A. L. de. (2020). Caracterização epidemiológica da hanseníase nas regiões Norte e Nordeste do Brasil. Research, Society and Development, 9(8): 1-9.

World Health Organization (WHO) (2019). Global leprosy update, 2018: moving towards a leprosy free world. Genebra. https://apps.who.int/iris/bitstream/handle/10665/326775/WER9435-36-en-fr.pdf?ua=1

World Health Organization. (2009). Enhanced global strategy for further reduce the disease burden due to leprosy (plan period 2011-2015). Regional Office for South-East Asia, New Delhi. https://apps.who.int/iris/handle/10665/205004 